\title{
Exotic Floristic Composition of Peddagattu, the proposed site for Uranium mining, Nalgonda district, Telangana state, India
}

\author{
A. Baleeshwar Reddy ${ }^{1^{*}}$, V. Hanumantha Rao ${ }^{2}$ V. Vasudeva rao ${ }^{2}$ \\ and A. Vijaya Bhasker Reddy ${ }^{1}$ \\ ${ }^{1}$ Department of Botany, Osmania University, Hyderabad-500 007, Telangana state, India. \\ ${ }^{2}$ AINP on Vertebrate Pest Management, Prof. Jayasanker Telangana state Agricultural University, \\ Rajendranagar, Hyderbad- 500030, Telangana state, India.
}

Corresponding author e-mail: baleeshwarreddy@gmail.com*

Keywords: Exotic species, Peddagattu, Tropical America, Uranium mining.

\begin{abstract}
The present investigation reflects the findings of exotic floristic composition at Peddagattu, Nalgonda district, Telangana State, India. A total of 125 exotic species of taxa belonging to 101 genera and 44 families were recorded in the study. Leguminosae is the most dominant family representing with 21 species, followed by Compositae (14 species), Apocynaceae, Convolvulaceae and Poaceae ( 7 species each). As per the nativity of exotic species representation in the study area are mostly from belongs to tropical America followed by tropical Africa and South America at Peddagattu.
\end{abstract}

\section{INTRODUCTION}

A flora refers to the 'Systematic enumeration of plants of a given region'. Flora is divided in to two categorized i.e. native flora and exotic flora. Species introduced into habitats where they are not native are referred as exotic species. Indo- Gangetic plains and the Thar Desert regions of India are rich in exotic flora while Himalayan and Peninsular regions are poor in exotic flora (Ahmad. 1999). Many exotic species have been purposefully introduced for food, fibber, erosion control, as agricultural crops, ornamental and landscape plants. Exotic species have tolerant of a wide range of soil and weather conditions, produce abundant amount of seed that disperse easily, grows insistent root systems, long flowering and fruiting periods. These are free from parasites, pathogens, predators and competitors. They can be thought of as biological pollutants are considered to be the most damaging agents of habitat alternation (Westbrooks, 1999). Infestations by exotic species may lead to great conservation concern (Wilcove et al. 1998). Invasive non- native species inflict harmful ecological and economic impacts upon ecosystems in non native regions (Pimentel et al., 2005; Meyeron and Mooney 2007). Several studies have been conducted to explore the exotic floristic composition of a particular region in India (Nagar et al., 2001; Tomar et al., 2008; Singh. 2011, 2012). The main objective of the present study is to explore the exotic flora of Peddagattu, Nalgonda district, Telangana state.

\section{MATERIALS AND METHODS}

Study area: Peddagattu in Nalgonda district (Telangana State) lies between 16.53889 and 16.72140 North latitude and 79.40643 and 79.09033 East longitude and covers $30 \mathrm{~km}$ radius (Fig. 1). The annual rainfall varies between 56 and $62 \mathrm{~cm}$ with the annual mean temperature of $34^{0} \mathrm{c}$. Physiographically Peddagattu consist of flat topped hills composed of proterozoic sediments. It is in close proximity to Nagarjunasagar canal system. The rocky exposures at the bottom of the hills generally are composed of granitic rocks. The general drainage pattern is dendritic indicating homogeneous nature of formations. The general elevation of the hills is about $300 \mathrm{~m}$ and the ground is $215 \mathrm{~m}$. Majority of the land area is under cultivation. The crops raised in the area are rice, sorghum, cotton and chilies along with sweet lime and mangoes orchards. 


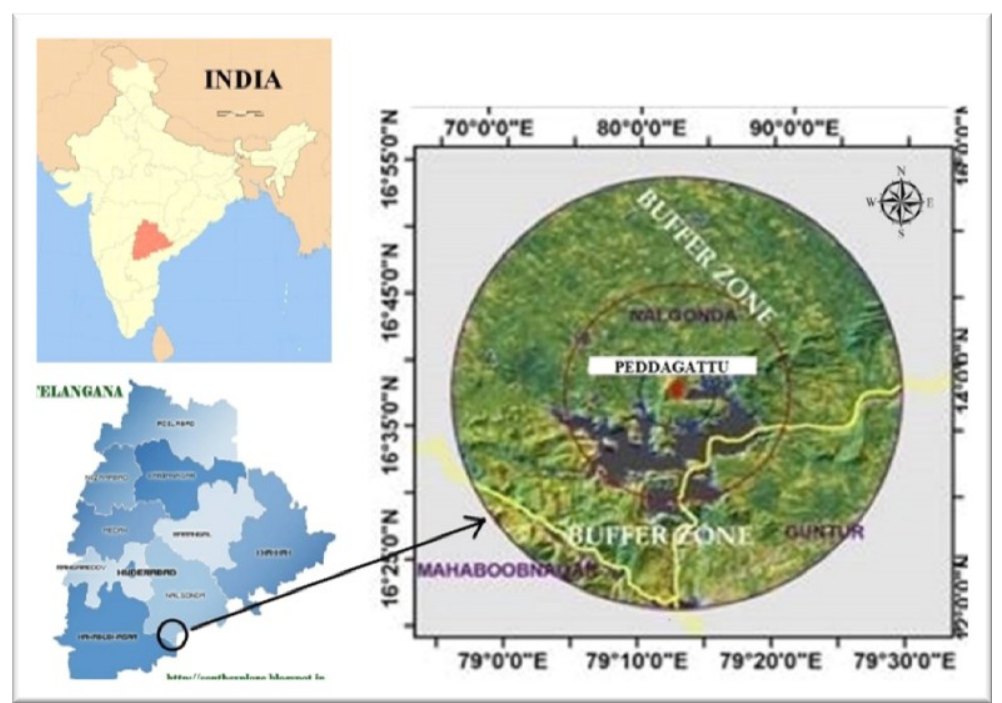

Figure. 1 Map of Study area

Methodology: The field work was carried out from April 2010 to December 2013. Floral exploration trips were regularly conducted on pre and post monsoon seasons to get the maximum list of exotic species in each habitat. The specimens were identified from their key vegetative and reproductive features using the Flora of Nalgonda district (Rao et al., 2001), Flora of Guntur district (Pullaiah et al., 2000) and Flora of the Presidency of Madras (Gamble and Fischer, 1915-1935). The plant species collected were processed and mounted on herbarium sheets. The native ranges of each species were recorded from Internet, Invasive Alien Flora of India (Reddy et al, 2008) and catalogue of invasive alien flora of India (Reddy .2008). The collected specimens were pressed and deposited at the AINP on Vertebrate Pest Management, Prof. Jayashanker Telangana State Agricultural Univrsity, Rajendranagar, Hyderabad.

\section{RESULTS AND DISCUSSION}

A total of 125 exotic species belonging to 101 genera and 44 families are reported from the study area (Table.1). Similar to the present study, Singh et al., (2012) reported, 198 exotic plant species in exotic floristic composition of the Varanasi district of Uttara Pradesh and also Tomar et al., (2008) reported 76 exotic species in flora of Baghpat district of Western Utter Pradesh. Habit wise analysis showed that herbs are predominant with 70 species followed by Trees (25), shrub (13) and Climbers (4). The family Leguminosae (21) contribution was maximum in number of exotic flora, followed by Compositae (14 species), Apocynaceae, Convolvulacea and Poacea with 7 species each. Contrary to the result of Present study, Singh et al., (2012) reported the dominance of Fabaceae, Asteracea and Poacaceae families of the exotic floristic Composition of the Varanasi district.

Representative of Tropical America exotic species composition was high in the study area with 47 species followed by representative of Tropical Africa and South America with 14 \&12 species respectively. The other regions such as Australia, Brazil, Madagascar, Mediterranean region, Mexico, Srilanka and West Indies representative are very low. Other studies also revealed at different locations the dominance of the flora of Tropical American origin in India (Reddy. 2008; Tomer et al., 2008; Singh, 2011, 2012). 


\section{CONCLUSION}

The present study shows that the area was rich in exotic species. The Leguminosae and Compositae are the dominant families of the study area. The flora of Tropical America origin dominates the exotic floristic compostion of the Peddagattu, Nalgonda district, Telanagna State, India.

Table. 1 Exotic floral of Peddagattu, Nalgonda district, Telangana State, India

\begin{tabular}{|c|c|c|c|c|}
\hline S. No & Scientific Name & Family & $\begin{array}{c}\text { Growth } \\
\text { form }\end{array}$ & Nativity \\
\hline 1 & Acacia auriculiformis Benth. & Leguminosae & Tree & Australia \\
\hline 2 & Acanthospermum hispidum DC. & Compositae & Herb & Brazil \\
\hline 3 & Agave americana $\mathrm{L}$. & Asparagaceae & Shrub & Central America \\
\hline 4 & Ageratum conyzoides (L.) L. & Compositae & Herb & Central America \\
\hline 5 & Albizia saman (Jacq.) Merr. & Leguminosae & Tree & $\begin{array}{l}\text { Mediterranean } \\
\text { region }\end{array}$ \\
\hline 6 & Aloe vera (L.) Burm.f. & Asparagaceae & Herb & Trop. America \\
\hline 7 & Alternanthera pungens Kunth & Amaranthaceae & Herb & Trop. America \\
\hline 8 & $\begin{array}{l}\text { Alternanthera sessilis (L.) } \\
\text { R.Br. ex DC. }\end{array}$ & Amaranthaceae & Herb & Trop. America \\
\hline 9 & Amaranthus spinosus L. & Amaranthaceae & $\begin{array}{l}\text { Under } \\
\text { shrub }\end{array}$ & America \\
\hline 10 & Anacardium occidentale L. & Anacardiaceae & Tree & $\begin{array}{c}\text { West Indies \& } \\
\text { Brazil }\end{array}$ \\
\hline 11 & Annona squamosa L. & Annonaceae & Tree & Trop. America \\
\hline 12 & $\begin{array}{l}\text { Antigonon leptopus Hook. \& } \\
\text { Arn }\end{array}$ & Polygoniaceae & Herb & South America \\
\hline 13 & Argemone mexicana L. & Papaveraceae & Herb & Ethiopia \\
\hline 14 & Asparagus racemosus Willd. & Asparagaceae & Climber & Srilanka \\
\hline 15 & Bauhinia purpurea L. & Leguminosae & Tree & West Indies \\
\hline 16 & Biden pilosa $\mathrm{L}$. & Compositae & Herb & Trop. America \\
\hline 17 & $\begin{array}{l}\text { Blainvillea acmella( } \\
\text { L)Philipson }\end{array}$ & Compositae & Herb & Trop. America \\
\hline 18 & Borassus flabellifer L. & Arecaceae & Tree & Trop. America \\
\hline 19 & $\begin{array}{l}\text { Calotropis gigantea }(\mathrm{L} .) \\
\text { Dryand. }\end{array}$ & Apocynaceae & Shrub & Trop. Africa \\
\hline 20 & $\begin{array}{l}\text { Calotropis procera (Aiton) } \\
\text { Dryand. }\end{array}$ & Apocynaceae & Shrub & Trop. Africa \\
\hline 21 & Canna indica L. & Cannaceae & Shrub & Africa \\
\hline 22 & Carica papaya $\mathrm{L}$. & Caricaceae & Tree & Mexico \\
\hline 23 & Carissa carandas L. & Apocynaceae & Shrub & South Africa \\
\hline 24 & Casuarina equisitifolia L. & Casuarinaceae & Tree & Australia \\
\hline 25 & $\begin{array}{l}\text { Catharanthus pusillus (Murray) } \\
\text { G.Don }\end{array}$ & Apocynaceae & Herb & Trop. America \\
\hline 26 & $\begin{array}{l}\text { Catharanthus roseus (L.) } \\
\text { G.Don }\end{array}$ & Apocynaceae & Herb & Madagascar \\
\hline 27 & Celosia argentea $\mathrm{L}$. & Amaranthaceae & Herb & Trop. Africa \\
\hline 28 & $\begin{array}{l}\text { Chamaecrista absus (L.) } \\
\text { H.S.Irwin \& Barneby }\end{array}$ & Leguminosae & Herb & Trop. America \\
\hline
\end{tabular}




\begin{tabular}{|c|c|c|c|c|}
\hline 29 & $\begin{array}{l}\text { Chamaecrista pumila (Lam.) } \\
\text { K.Larsen }\end{array}$ & Leguminosae & Herb & Trop. America \\
\hline 30 & Chloris barbata Sw. & Poaceae & Herb & Trop. America \\
\hline 31 & $\begin{array}{l}\text { Chrozophora rottleri (Geis.) } \\
\text { Spreng. }\end{array}$ & Euphorbiaceae & Herb & Trop. Africa \\
\hline 32 & Cleome gynandra L. & Cleomaceae & Herb & Trop. America \\
\hline 33 & Cleome monophylla L. & Cleomaceae & Herb & Trop. Africa \\
\hline 34 & Cleome viscosa $\mathrm{L}$. & Cleomaceae & Herb & Trop. America \\
\hline 35 & Corchoru aestuans L. & Malvaceae & Herb & Trop. America \\
\hline 36 & Corchorus trilocularis L. & Malvaceae & Herb & Trop. Africa \\
\hline 37 & Corchorus tridens L. & Malvaceae & Herb & Trop. Africa \\
\hline 38 & Croton bonplandianus Baill. & Euphorbiaceae & Herb & South America \\
\hline 39 & Cryptostegia grandiflora R.Br. & Apocynaceae & Shrub & Trop. America \\
\hline 40 & Cuscuta chinensis Lam. & Convolvulaceae & Parasite & Mediterranean \\
\hline 41 & Cynodon dactylon (Linn.) Pers & Poaceae & Herb & Trop. America \\
\hline 42 & Cyperus rotundus L. & Cyperaceae & Herb & Eurasia \\
\hline 43 & Cyperus difformis L. & Cyperaceae & Herb & Trop. America \\
\hline 44 & Cyperus iria $\mathrm{L}$. & Cyperaceae & Herb & Trop. America \\
\hline 45 & Datura innoxia Mill. & Solanaceae & Shrub & Trop. America \\
\hline 46 & Datura metel L. & Solanaceae & Herb & Trop. America \\
\hline 47 & Delonix regia (Hook.) Raf. & Leguminosae & Tree & Madagascar \\
\hline 48 & Dicoma tomentosa Cass & Compositae & Herb & Trop. Africa \\
\hline 49 & Digera muricata (L.) Mart. & Amaranthaceae & Herb & SW Asia \\
\hline 50 & Dinebra retroflexa (Vahl)Panz & Poaceae & Herb & Trop. America \\
\hline 51 & Echinochloa colona (L.) Link & Poaceae & Herb & Trop. S. America \\
\hline 52 & $\begin{array}{l}\text { Echinochloa crusgalli (L) } \\
\text { Beauv }\end{array}$ & Poaceae & Herb & Trop. S. America \\
\hline 53 & Echinops echinatus Roxb. & Compositae & Herb & Afghanistan \\
\hline 54 & Eclipta prostrata (L.) L. & Compositae & Herb & South America \\
\hline 55 & Eichornia crassipes Solms & Pontederiaceae & Hydrophyte & Brazil \\
\hline 56 & $\begin{array}{l}\text { Eragrostis tenella (L.) Beauv. } \\
\text { \& Schult. }\end{array}$ & Poaceae & Herb & Africa \\
\hline 57 & Eucalyptus globulus Labill. & Myrtaceae & Tree & Australia \\
\hline 58 & Euphorbia heterophylla L. & Euphorbiaceae & Herb & Trop. America \\
\hline 59 & Euphorbia hirta L. & Euphorbiaceae & Herb & Trop. America \\
\hline 60 & $\begin{array}{l}\text { Euphorbia pulcherrima Willd. } \\
\text { ex Klotzsch }\end{array}$ & Euphorbiaceae & Herb & Mexico \\
\hline 61 & Gomphrena serrata L. & Amaranthaceae & Herb & Trop. America \\
\hline 62 & $\begin{array}{l}\text { Grangea maderaspatana (L.) } \\
\text { Desf. }\end{array}$ & Compositae & Herb & Trop. S. America \\
\hline 63 & Heliotropium indicum L. & Boraginaceae & Herb & South America \\
\hline 64 & $\begin{array}{l}\text { Holoptelea grandis (Hutch.) } \\
\text { Mildbr. }\end{array}$ & Ulmaceae & Tree & Pacific Islands \\
\hline 65 & Hyptis suaveolens (L.) Poit. & Lamiaceae & Herb & Trop. America \\
\hline 66 & Indigofera astragalina DC. & Leguminosae & Herb & Trop. America \\
\hline 67 & Indigofera linifolia (L.f.) Retz. & Leguminosae & Herb & Trop. Africa \\
\hline
\end{tabular}




\begin{tabular}{|c|c|c|c|c|}
\hline 68 & Ipomoea carnea Jacq. & Convolvulaceae & Shrub & Trop. America \\
\hline 69 & Ipomoea hederifolia L. & Convolvulaceae & Herb & Trop. America \\
\hline 70 & Ipomoea pes-tigridis L. & Convolvulaceae & Herb & Trop. E. Africa \\
\hline 71 & Ipomoea quamoclit L. & Convolvulaceae & Climber & Trop. America \\
\hline 72 & $\begin{array}{l}\text { Ipomoea obscura (L.) Ker } \\
\text { Gawl. }\end{array}$ & Convolvulaceae & Herb & Trop. Africa \\
\hline 73 & Jatropha curcas L. & Euphorbiaceae & Shrub & Trop. America \\
\hline 74 & Lagascea mollis Cav. & Compositae & Herb & Trop. C. America \\
\hline 75 & Lantana camara $\mathrm{L}$. & Verbenaceae & Shrub & America \\
\hline 76 & Lawsonia inermis L. & Lythraceae & Tree & Northern Africa \\
\hline 77 & Leonotis nepetifolia (L.) R.Br. & Lamiaceae & Herb & Trop. Africa \\
\hline 78 & $\begin{array}{l}\text { Leucaena leucocephala (Lam.) } \\
\text { de Wit }\end{array}$ & Leguminosae & Tree & Trop. America \\
\hline 79 & Ludwigia adscendens (L.) Hara & Onagraceae & Herb & Trop. America \\
\hline 80 & Ludwigia perennis L. & Onagraceae & Herb & Trop. Africa \\
\hline 81 & Manilkara zapota (L.) P.Royen & Sapotaceae & Tree & South America \\
\hline 82 & Martynia annua L. & Martyniaceae & Shrub & Mexico \\
\hline 83 & Merremia aegyptia (L.) Urb. & Convolvulaceae & Herb & Trop. America \\
\hline 84 & Mimosa pudica L. & Leguminosae & Herb & Brazil \\
\hline 85 & Opuntia elatior Mill. & Cactaceae & Shrub & South America \\
\hline 86 & Oxalis corniculata L. & Oxalidaceae & Herb & Northern America \\
\hline 87 & Parkinsonia aculeata L. & Leguminosae & Tree & Mexico \\
\hline 88 & Parthenium hysterophorus L. & Compositae & Herb & Trop. America \\
\hline 89 & Passiflora foetida $\mathrm{L}$. & Passifloraceae & Climber & South America \\
\hline 90 & Pedalium murex L. & Pedaliaceae & Herb & Trop. America \\
\hline 91 & $\begin{array}{l}\text { Peltophorum pterocarpum } \\
\text { (DC.) K.Heyne }\end{array}$ & Leguminosae & Tree & $\begin{array}{c}\text { Srilanka \& } \\
\text { Malaysia } \\
\end{array}$ \\
\hline 92 & Phoenix sylvestris (L.) Roxb. & Arecaceae & Tree & West Asia \\
\hline 93 & Physalis angulataL. & Solanaceae & Herb & South America \\
\hline 94 & Pistia stratiotes L. & Araceae & Herb & Trop. America \\
\hline 95 & $\begin{array}{l}\text { Pithecellobium dulce (Roxb.) } \\
\text { Benth. }\end{array}$ & Leguminosae & Tree & $\begin{array}{l}\text { Central \& South } \\
\text { America }\end{array}$ \\
\hline 96 & Plumeria rubra L. & Apocynaceae & Tree & South America \\
\hline 97 & Portulaca oleracea L. & Portulacaceae & Herb & $\begin{array}{c}\text { Northen Africa \& } \\
\text { Europe }\end{array}$ \\
\hline 98 & $\begin{array}{l}\text { Prosopis julifera(Molina) } \\
\text { Stuntz }\end{array}$ & Leguminosae & Tree & Central America \\
\hline 99 & Psidium guajava L. & Myrtaceae & Shrub & South America \\
\hline 100 & Punica granatum L. & Lythraceae & Tree & Iran, Afghanistam \\
\hline 101 & Ruellia tuberosa L. & Acanthaceae & Herb & Trop. America \\
\hline 102 & Saccharum spontaneum L. & Poaceae & Herb & Trop. W. Asia \\
\hline 103 & Scoparia dulcis L. & Plantaginaceae & Herb & Trop. America \\
\hline 104 & $\begin{array}{l}\text { Senna obtusifolia (L.) } \\
\text { H.S.Irwin \& Barneby }\end{array}$ & Leguminosae & $\begin{array}{l}\text { Under } \\
\text { Shrub }\end{array}$ & Trop. America \\
\hline 105 & Senna occidentalis (L.) Link & Leguminosae & $\begin{array}{l}\text { Under } \\
\text { Shrub }\end{array}$ & Trop. America \\
\hline
\end{tabular}




\begin{tabular}{|c|l|c|c|c|}
\hline 106 & Senna siamea Lam. & Leguminosae & Tree & Myanmar \\
\hline 107 & Senna tora (L.) Roxb. & Leguminosae & $\begin{array}{c}\text { Under } \\
\text { shrub }\end{array}$ & South America \\
\hline 108 & $\begin{array}{l}\text { Senna uniflora } \text { (Mill.) } \\
\text { H.S.Irwin \& Barneby }\end{array}$ & Leguminosae & Herb & $\begin{array}{c}\text { Trop. South } \\
\text { America }\end{array}$ \\
\hline 109 & Sida acuta Burm.f. & Malvaceae & Herb & Trop. America \\
\hline 110 & Solanum americanum L. & Solanaceae & Herb & Trop. America \\
\hline 111 & Sonchus oleraceus (L.) L. & Compositae & Herb & Mediterranean \\
\hline 112 & $\begin{array}{l}\text { Spermacoce } \text { neohispida } \\
\text { Govaerts }\end{array}$ & Rubiaceae & Herb & Mediterranean \\
\hline 113 & Sphaeranthus indicus Linn. & Compositae & Herb & Africa \\
\hline 114 & Tamarindus indica L. & Leguminosae & Tree & Trop. Africa \\
\hline 115 & $\begin{array}{l}\text { Tecoma stans } \text { (L.) Juss. ex } \\
\text { Kunth }\end{array}$ & Bignoniaceae & Tree & America \\
\hline 116 & Terminalia catappa L. & Combretaceae & Tree & Malaysia \\
\hline 117 & Tribulus terrestris L. & Zygophyllaceae & Herb & Trop. America \\
\hline 118 & Tridax procumbens (L.) L. & Compositae & Herb & Mexico \\
\hline 119 & Triumfetta pentandra Rich. & Malvaceae & Herb & Trop. America \\
\hline 120 & Typha domingensis Pers. & Typhaceae & Herb & Trop. America \\
\hline 121 & Urena lobata L. & Malvaceae & Shrub & Trop. Africa \\
\hline 122 & Waltheria indica L. & Malvaceae & Herb & Trop. America \\
\hline 123 & Withania somnifera (L.) Dunal & Solanaceae & Herb & Mediterranean \\
\hline 124 & Xanthium strumariumium L. & Compositae & Herb & Trop. America \\
\hline 125 & Ziziphus Jujuba Mill. & Rhamnaceae & Tree & China \\
\hline
\end{tabular}

\section{ACKNOWLEDGMENT}

The authors are grateful to Board of Research on Nuclear science (BRNS), BARC, Government of India, for funding the project. We also extended our thanks to Prof. Jayasanker Telangana state Agricultural University (Formerly under Acharya. N. G. Ranga Agricultural University) for granting permission to conduct studies at Peddagattu, Nalgonda district of Telangana State.

\section{References}

[1] Ahmad A (1999). India: A general Geography. (N.C.E.R.T. Publications, New Delhi, Indi) 6676.

[2] Gamble, J.S. and C. E. C. Fischer. (1915-1935). Flora of the Presidency of Madras (repr. ed. 1957. Calcutta).

[3] Meyrson LA, Mooney HA (2007) Invasive alien species in an era of globalization. Frontiers in Ecology and Environment 5(4): 199-208

[4] Nagar PS, SJ Pathak and SM Pandya (2004). The alien flora of the Barda hills and surroundings in Gujarat, India. Indian Journal of Forestry 27(1)25-38.

[5] Pimentel D, Zuniga R, Morrison D (2005) Update on the environmental and economic costs associated with alien invasive species in the United States. Ecological Economics 52: 273-288, http: dx.doi.org/10.10.1016/j.ecolecon.2004.10.002

[6] Pullaiah,T. Ramakrishnaiah V. Sadhya Rani, S. and Rao, P. N. (2000). Flora of Guntur district, Andhra Pradesh, India. Regency Publications. 
[7] Rao, P.N. Raghava Swamy, B.V and Pullaiah T. (2001). Flora of Nalgonda district Andhra Pradesh, India. ShipraPublications.

[8] Reddy C.S., Bagyannarayana G., Reddy K.N and Raju V.S. (2008). Invasive Alien Flora of India.

[9] Reddy C.S., Catalogue of Invasive alien species flora of India. (2008). Life science journal 5(2): 85-87

[10] Singh A (2011). Exotic flora of the Banaras Hindu University main campus, India. Journal of Ecology and the Natural Environment 3(10)337-343.

[11] Singh A (2012). Exotic flora of the Chandauli district Utter Pradesh, India: An Overview. India Journal of Forestry 35 (1)79-89.

[12] Tomar A, H Singh and V Singh (2008). Exotic elements in the flora of Baghpat district, Utter Pradesh. Indian Journal of Forestry 31(3) 463-471.

[13] Westbrooks R (1991). Plant protection issues I. A commentary on new weeds in the United States. Weed Technology 5 232-237.

[14] Wilcove Ds, Rothstein D, Dubow J, Phillips A, Losos E (1998) Quantifying threats to imperiled species in the United States. BioScience 48: 607-615. http://dx.doi.org/10.2307/1313420. 